\title{
DISPERSION RELATIONS IN BILAYER GRAPHENE AT FINITE TEMPERATURE
}

\author{
Nguyen Van Men ${ }^{a^{*}}$ \\ ${ }^{a}$ The Faculty of Education, An Giang University, Vietnam National University Ho Chi Minh City, \\ An Giang, Vietnam \\ *Corresponding author: Email: nvmen@agu.edu.vn
}

\author{
Article history \\ Received: April 22nd 2021 \\ Received in revised form: June $12^{\text {th }}, 2021 \mid$ Accepted: June $24^{\text {th }}, 2021$ \\ Available online: July $22^{\text {nd }}, 2021$
}

\begin{abstract}
It is well-known that material technology is considered as one of the scientific fields attracting a lot of attention from scientists. Recently, graphene, a perfect two-dimensional structure, has attracted a large amount of interest from researchers due to its unique properties and possible applications in a variety of technological fields. The dispersion relations in graphene demonstrate that this material can be used to create plasmonic devices with potentially more features and less energy consumption than recent semiconductors. This paper calculates the dispersion relations in a bilayer graphene structure at finite temperatures using the random-phase approximation. The numerical results show that as temperature increases from zero, the plasmon frequency decreases slightly near the Dirac points and then increases noticeably. In large wave vector regions, the plasmon frequency behaves as an increasing function of temperature. The contribution of carrier density to plasmon frequency in the bilayer graphene system diminishes when temperature effects are taken into account. We observed that temperature significantly affects the dispersion relations in bilayer graphene systems; therefore, this factor should not be neglected in efforts to improve models or in comparisons with experimental results.
\end{abstract}

Keywords: Bilayer graphene; Dispersion relations; Dynamical dielectric function; Finite temperature; Random-phase approximation.

DOI: http://dx.doi.org/10.37569/DalatUniversity.11.4.882(2021)

Article type: (peer-reviewed) Full-length research article

Copyright () 2021 The author(s).

Licensing: This article is licensed under a CC BY-NC 4.0 


\section{INTRODUCTION}

Graphene, a perfect two-dimensional structure consisting of one layer of carbon atoms arranged in a honeycomb lattice, has recently attracted a large amount of attention from scientists due to its unique electronic and optical properties as well as for its technological applications compared to previous materials. Theoretical studies on monolayer graphene (MLG) show that quasi-particles in MLG behave as massless fermions with linear low-energy dispersion and a zero-band gap. This behavior differs completely from the parabolic dispersion and finite gap in conventional twodimensional electron gas (2DEG) systems. Due to these differences, graphene applications exist in a variety of technological fields, such as photonics, optoelectronics, energy storage, and other areas (Alonzo-González et al., 2017; Das Sarma et al., 2010; Das Sarma et al., 2011; Jiang et al., 2017; Ju et al., 2011; Lundeberg et al., 2017; Ni et al., 2016; Ni et al., 2018; Sunku et al., 2018).

Bilayer graphene (BLG), a structure consisting of two parallel MLG sheets at sufficiently small separation, is viewed as an ideal two-dimensional system. The strong coupling between electrons in the two MLG sheets in BLG makes quasi-particles in BLG systems become chiral massive fermions with parabolic dispersion in the lowenergy limit. The difference in the characteristics of the quasi-particles leads to differences in the polarizability, dynamical dielectric function, screening properties, and dispersion relations of BLG systems compared to MLG and ordinary 2DEG structures (Das Sarma et al., 2010; Das Sarma et al., 2011; Sensarma et al., 2010).

Collective excitations in materials have been studied for a long time and have been applied to create plasmonic devices. The dispersion relations in MLG at zero and finite temperature have been investigated with many interesting suggestions for applications in a variety of technological fields (Hwang \& Das Sarma, 2007; Politano et al., 2016; Politano et al., 2017; Ramezanali et al., 2009; Yan et al., 2012). Several studies of collective excitations in multilayer graphene structures have been published (Dong \& Nguyen, 2019; Wachsmuth et al., 2014; Zhu et al., 2013). Wang \& Chakraborty (2007) investigated Coulomb screening and plasmon modes in undoped graphene systems and demonstrated some interesting results. According to their paper, one weakly damped plasmon mode is found in these systems in the case of finite temperature. In 2010, the screening and collective excitations in doped BLG at zero temperature were studied and published by Sensarma and his co-workers for the first time (Sensarma et al., 2010). The calculations for collective excitations in layered structures consisting of BLG have been studied intensively (Nguyen et al., 2019a, 2019c, 2021; Nguyen \& Dong, 2018). However, recent publications have neglected the effects of temperature, although previous papers have observed significant temperature effects on the plasmon properties of layered structures (Patel, 2015; Nguyen et al., 2019b; Ramezanali et al., 2009; Dinh \& Nguyen, 2013; Vazifehshenas et al., 2010). To our knowledge, no calculations have been performed to date for the plasmon properties of BLG at finite temperature. This paper provides calculations for the dispersion relations in BLG taking into account temperature effects to improve the model and the agreement between theoretical and experimental results. As observed in previous 
publications, the random-phase approximation leads to good results for high-carrier density systems such as graphene (Das Sarma et al., 2010; Hwang \& Das Sarma, 2007, 2009; Sensarma et al., 2010), therefore this approximation is used to calculate the dynamical dielectric function of the BLG structure in this paper.

\section{THEORETICAL APPROACH}

We investigate the dispersion relations in a BLG system on the dielectric substrate $\mathrm{SiO}_{2}$ (with $\kappa_{\mathrm{SiO}_{2}}=3.8$ ). Temperature effects are taken into account in all calculations. Note that BLG systems considered in this paper have parabolic low-energy dispersion. In this approximation, the Hamiltonian of BLG has the form (Das Sarma et al., 2010; Sensarma et al., 2010):

$$
\widehat{H}_{2, x y}=-\frac{1}{2 m}\left(\begin{array}{cc}
0 & \left(k_{x}-i k_{y}\right)^{2} \\
\left(k_{x}+i k_{y}\right)^{2} & 0
\end{array}\right)
$$

where $m=0.033 m_{0}$ is the effective mass, and $m_{0}$ is the vacuum mass of electrons. For the Hamiltonian in Equation (1), the energy and the wave function are

$$
E= \pm \frac{k^{2}}{2 m}, \quad \psi_{ \pm}=\frac{1}{\sqrt{2}}\left(\begin{array}{c}
0 \\
\mp e^{i 2 \varphi}
\end{array}\right) e^{i \vec{k} \vec{r}} .
$$

In Equation (2), the upper and lower signs correspond to the conduction and valence bands, respectively, and $\varphi$ is the argument of the wave vector in the graphene plane $\vec{k}=\left(k_{x}, k_{y}\right)=k(\cos \varphi, \sin \varphi)$.

It has been proven that the dispersion relations of the system can be determined from the zeroes of the temperature-dependent dynamical dielectric function (Nguyen \& Nguyen, 2018; Nguyen et al., 2019a; Nguyen \& Dong, 2019; Dinh \& Nguyen, 2013; Vazifehshenas et al., 2010; Zhu et al., 2013):

$$
\varepsilon\left(q, \omega_{p}-i \gamma, T\right)=0 .
$$

Here $\omega_{p}$ is the plasmon frequency at a given momentum $q$, and $\gamma$ is the damping rate of the respective plasma oscillations. In the case of weak damping, the solutions of Equation (3) can be found approximately from the zeroes of the real part of the temperature-dependent dynamical dielectric function (Nguyen \& Nguyen, 2018; Nguyen et al., 2019a; Nguyen \& Dong, 2019; Dinh \& Nguyen, 2013; Vazifehshenas et al., 2010; Zhu et al., 2013)

$$
\operatorname{Re} \varepsilon\left(q, \omega_{p}, T\right)=0 .
$$

Within the random-phase approximation, the temperature-dependent dynamical dielectric function of the BLG system is

$$
\varepsilon(q, \omega, T)=1-v(q) \Pi(q, \omega, T)
$$


where $v(q)=2 \pi e^{2} /(\kappa q)$ is the Coulomb bare interaction in momentum space and $\kappa$ is the average permittivity of the substrate $\left(\mathrm{SiO}_{2}, \kappa_{\mathrm{SiO}_{2}}=3.8\right)$ and the air $\left(\kappa_{\text {air }}=1.0\right) . \Pi(q, \omega, T)$ is the polarizability function of BLG at finite temperature (Das Sarma et al., 2010; Das Sarma et al., 2011; Sensarma et al., 2010):

$$
\Pi(q, \omega, T)=g \sum_{\lambda, \lambda^{\prime}, \vec{k}}\left|g_{\vec{k}}^{\lambda, \lambda^{\prime}}(\vec{q})\right|^{2} \frac{f\left(E_{\vec{k}+\vec{q}}^{\lambda^{\prime}}\right)-f\left(E_{\vec{k}}^{\lambda}\right)}{\omega+E_{\vec{k}+\vec{q}}^{\lambda^{\prime}}-E_{\vec{k}}^{\lambda}+i \eta}
$$

Here $\left|g_{\vec{k}}^{\lambda, \lambda^{\prime}}(\vec{q})\right|^{2}=\left[1+\lambda \lambda^{\prime} \cos 2\left(\theta_{\vec{k}}-\theta_{\vec{k}+\vec{q}}\right)\right] / 2$ is the overlap wave function, $g=4$ denotes the degeneracy (spin and valley) factor, and $\lambda= \pm 1$ corresponds to the conduction and valence bands, respectively. The Fermi-Dirac contribution, $f(x)$, is formed as

$$
f(x)=\left(e^{\frac{x-\mu}{k_{B} T}}+1\right)^{-1} .
$$

In Equation (7), $\mu$ is the chemical potential. Detailed calculations have proven that in BLG systems $\mu=E_{F}$, which is the Fermi energy of BLG (Das Sarma et al., 2010; Das Sarma et al., 2011; Lv \& Wan, 2010; Sensarma et al., 2010).

The numerical solutions for Equation (4) with the temperature-dependent dynamical dielectric function in Equation (5) and the polarizability in Equation (6) show the dispersion relations for BLG systems.

\section{NUMERICAL RESULTS AND DISCUSSION}

In this section, we present numerical calculations for dispersion relations in BLG systems taking into account temperature effects. Note that $E_{F}, k_{F}$, and $T_{F}$ denote the Fermi energy, the Fermi wave vector, and the Fermi temperature of the structure, respectively. At the fixed carrier density $n=10^{12} \mathrm{~cm}^{-2}$, the Fermi wave vector of BLG is $k_{F}=\sqrt{n \pi} \approx 1.77 \times 10^{6} \mathrm{~cm}^{-2}$, corresponding to a Fermi energy of $E_{F} \approx 36.3 \mathrm{meV}$ and a Fermi temperature of $T_{F}=E_{F} / k_{B} \approx 421 K$.

Figure 1 presents dispersion relations in a BLG system with carrier density $n=10^{12} \mathrm{~cm}^{-2}$ plotted for $T=0, T=0.1 T_{F}, T=0.2 T_{F}$, and $T=0.5 T_{F}$. Figure 1a shows that the plasmon frequency decreases slightly with temperature in the small wave vector region (as seen in the inset), near the Dirac points and increases substantially with temperature in the larger wave vector region. The reason is that at higher temperature, electrons with large kinetic energy can be more easily excited, leading to larger-energy collective excitations. Similar behavior has been found in previous calculations of the dispersion relations in MLG structures (Nguyen et al., 2019b; Nguyen et al., 2020; Dinh \& Nguyen, 2013; Vazifehshenas et al., 2010). In the longwavelength region, the branches for $T=0$ (thick solid line) and $T=0.2 T_{F}$ (thin solid line) appear identical and only diverge in the larger wave vector region $\left(q \geq 0.3 k_{F}\right)$. As temperature increases noticeably, the plasmon frequency strongly increases, as seen 
from Figure $1 \mathrm{~b}$. The plasmon branches separate at a quite small wave vector, about $0.05 k_{F}$. The difference in plasmon frequency at a given wave vector between the two cases of finite temperature $\left(T=0.1 T_{F}\right.$ and $\left.T=0.5 T_{F}\right)$ is approximately $20 \%$ at the wave vector $q \approx 0.5 k_{F}$. For comparison, we plot in Figure $1 \mathrm{c}$ and $1 \mathrm{~d}$ the dispersion relations in MLG for the same parameters. The figures show that, in the small wave vector regions, temperature affects the collective excitations in MLG more strongly than in BLG systems. By contrast, in the larger wave vector regions, the dispersion relations increase more strongly in BLG than in MLG structures. Similar to other layered structures (Nguyen et al., 2019b; Ramezanali et al., 2009; Dinh \& Nguyen, 2013; Vazifehshenas et al., 2010), the effects of temperature on the plasmon modes in BLG systems are significant and should not be neglected, as found clearly in the large wave vector regions.

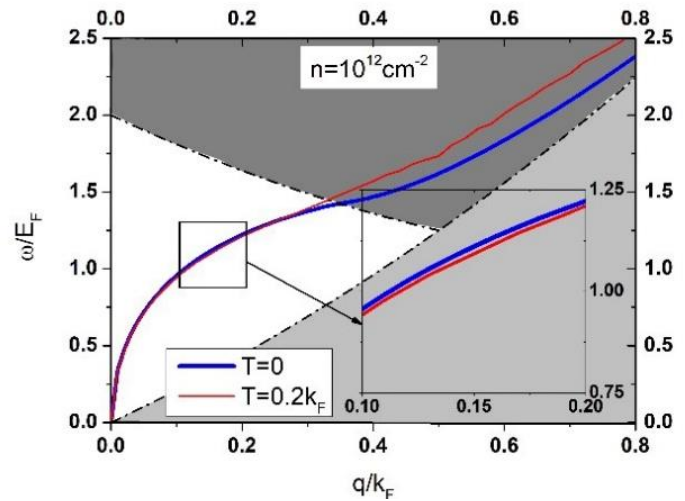

(a)

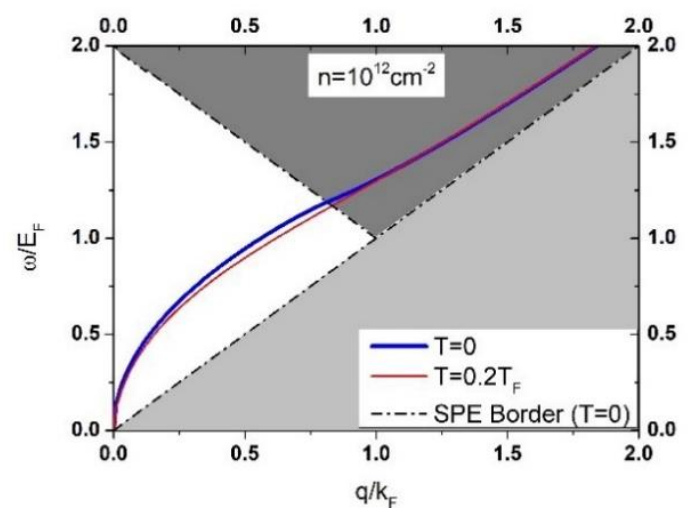

(c)

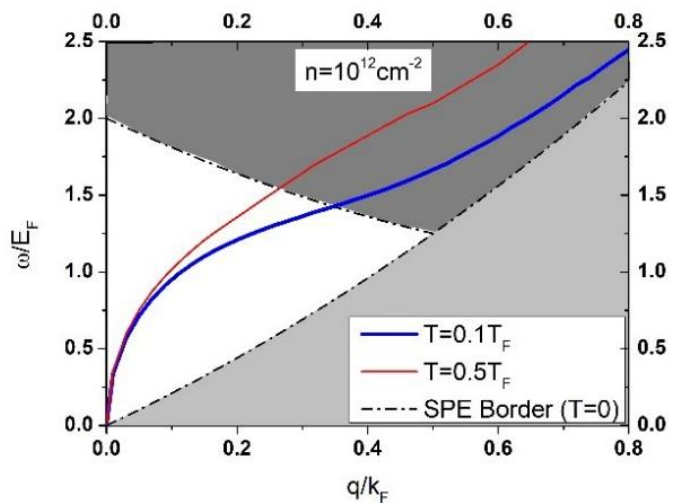

(b)

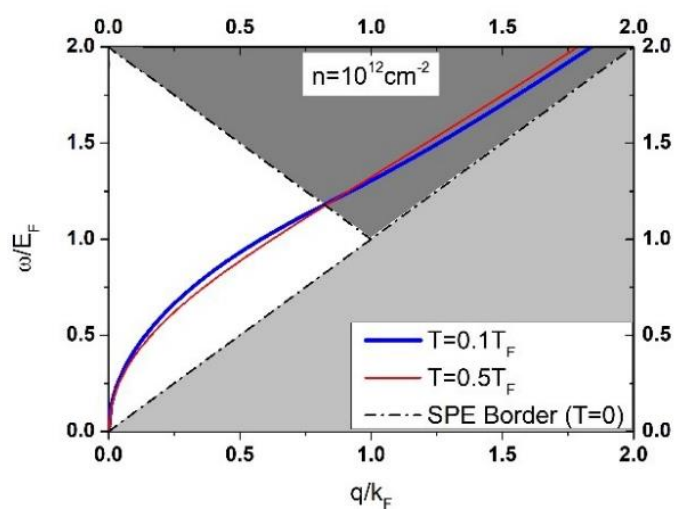

(d)

Figure 1. The dispersion relations in BLG (a), (b) and MLG (c), (d) systems with carrier density $n=10^{12} \mathrm{~cm}^{-2}$ for temperatures of $T=0, T=0.1 T_{F}, T=0.2 T_{F}$, and $T=0.5 T_{F}$

Note: The shaded regions denote the single-particle excitation (SPE) areas at zero temperature.

To study the effects of temperature on plasmon properties in detail Figure 2 plots the plasmon frequency in BLG and MLG systems as a function of temperature for several wave vectors and for carrier densities $n=10^{12} \mathrm{~cm}^{-2}$ (a), (c) and $n=10^{13} \mathrm{~cm}^{-2}$ 
(b). As seen in Figure 2a for $q=0.1 k_{F}$ and $q=0.3 k_{F}$, the plasmon frequency decreases slightly as the temperature increases slightly from zero, then increases significantly with temperature at larger temperatures. In the case of a larger wave vector $\left(q=0.4 k_{F}\right)$, the plasmon frequency behaves as an increasing function of the investigated temperature. This result shows good agreement with that demonstrated in Figure 1. Similar behavior for collective excitations in MLG and structures consisting of MLG has been obtained in previous papers (Nguyen et al., 2019b; Dinh \& Nguyen, 2013) and is seen in Figure 2c. The difference is that the decrease in the plasmon frequency in the small wave vector region of MLG systems is more noticeable than that in the other case. The pattern of plasmon curves for $n=10^{12} \mathrm{~cm}^{-2}$ (Figure 2a) differs only slightly compared to that of $n=10^{13} \mathrm{~cm}^{-2}$ (Figure 2b), although the carrier density is increased by ten times. However, the decrease in the plasmon frequency in the small wave vector region is extremely difficult to recognize. In other words, the increase in doping density in the BLG system reduces the effects of temperature on dispersion relations in BLG systems.

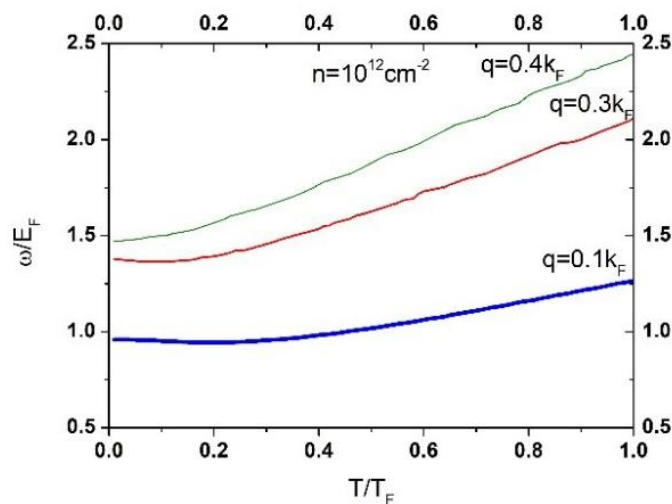

(a)

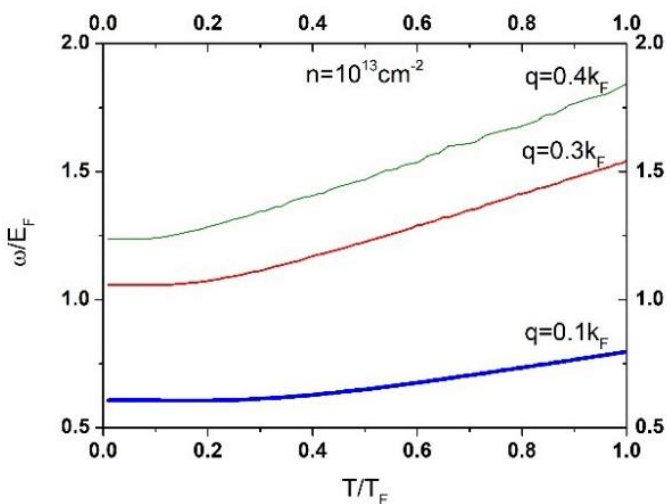

(b)

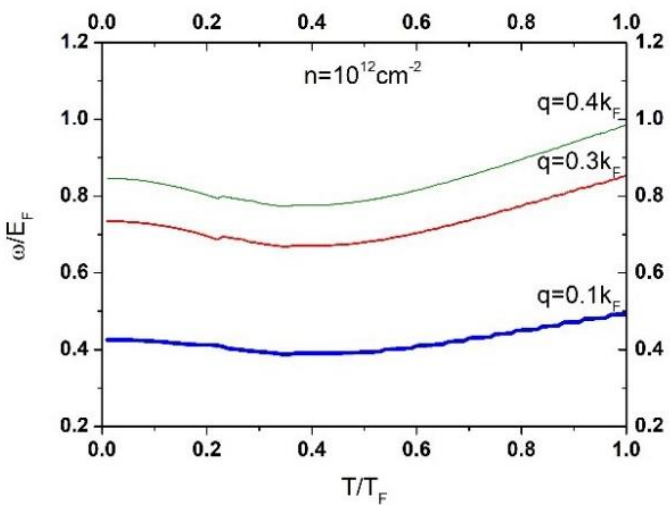

(c)

Figure 2. Plasmon frequency in BLG (a), (b) and MLG (c) systems as a function of temperature for wave vectors $q=0.1 k_{F}, q=0.3 k_{F}$, and $q=0.4 k_{F}$ plotted for carrier densities $n=10^{12} \mathrm{~cm}^{-2}$ (a), (c) and $n=10^{13} \mathrm{~cm}^{-2}$ (b)

Finally, we consider the effects of doping density on plasmon properties in BLG systems at finite temperature in comparison with the zero-temperature case. In Figure 3, 
we present the collective excitations in a BLG structure at $T=0.2 T_{F}$ and $T=0$ for doping densities $n=10^{12} \mathrm{~cm}^{-2}$ and $n=10^{13} \mathrm{~cm}^{-2}$. As seen from the figures, increased doping density strongly decreases the plasmon energy in both cases. This behavior comes from the dependence of the interaction parameter $\left(r_{s}\right)$ on the doping carrier density. In the BLG system, $r_{s} \sim n^{-1 / 2}$ decreases with the increase in doping density. Therefore, the increase in doping density leads to a decrease in plasmonic energy (Das Sarma et al., 2010; Das Sarma et al., 2011; Hwang \& Das Sarma, 2007; Sensarma et al., 2010). In the case of finite temperature, the plasmon frequency depends on the carrier density more weakly than in the zero-temperature case. Thus, taking temperature effects into account in the calculations diminishes the effect of doping density on plasmon properties in BLG systems.

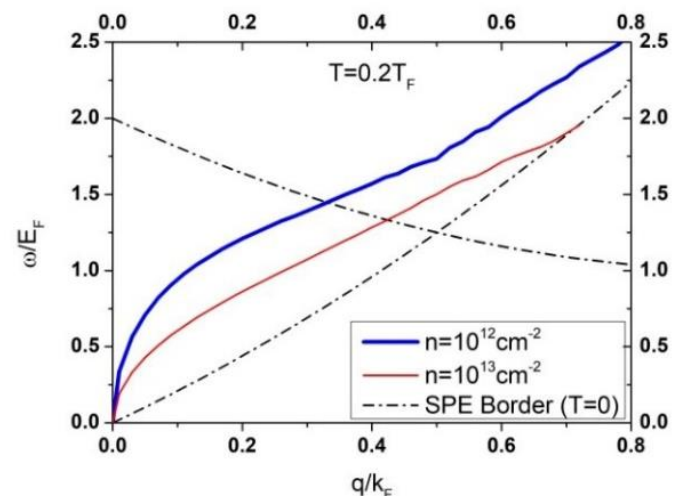

(a)

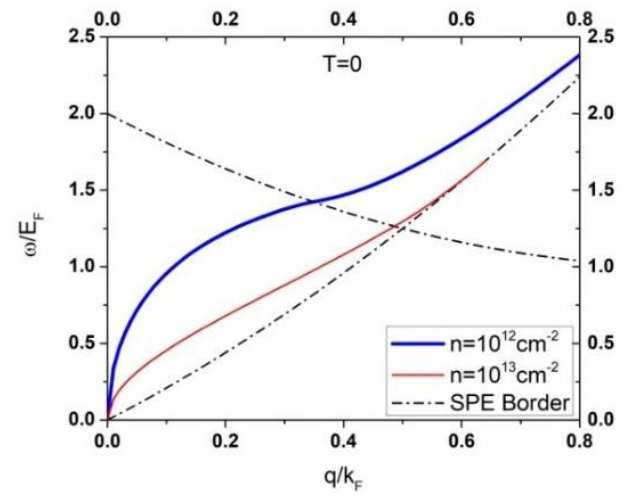

(b)

Figure 3. Dispersion relations in a BLG system at temperature $T=0.2 T_{F}$ and $T=0$ with doping densities $n=10^{12} \mathrm{~cm}^{-2}$ and $n=10^{13} \mathrm{~cm}^{-2}$

Note: Dash-dotted lines show the SPE boundary at zero temperature.

\section{CONCLUSION}

In summary, we have calculated the dispersion relations in BLG structures using the random-phase approximation at finite temperature. Numerical results show that the dependence of plasmon frequency on temperature in the small wave vector region is significantly different from that in the large wave vector region. Plasmon frequency noticeably increases with temperature in the latter, whereas it decreases slightly as the temperature increases from zero in the efficiently small wave vector region. Moreover, increased carrier density in BLG systems substantially decreases plasmon frequency, and the dispersion relations depend more weakly on increasing temperature. We observe that the collective excitations in BLG systems, when temperature effects are taken into account, are remarkably different from those at zero temperature, as found in previous publications. Our results may be useful in improving models and in helping material scientists develop technological applications for graphene. 


\section{ACKNOWLEDGMENT}

This research is funded by Vietnam National Foundation for Science and Technology Development (NAFOSTED) under grant number 103.01-2020.11.

\section{REFERENCES}

Alonzo-González, P., Nikitin, A. Y., Gao, Y., Woessner, A., Lundeberg, M. B., Principi, A., Forcellini, N., Yan, W., Vélez, S., Huber, A. J., Watanabe, K., Taniguchi, T., Casanova, F., Hueso, L. E., Polini, M., Hone, J., Koppens, H. L., \& Hillenbrand, R. (2017). Acoustic terahertz graphene plasmons revealed by photocurrent nanoscopy. Nature Nanotechnology, 12, 31-35.

Das Sarma, S., Hwang, E. H., \& Rossi, E. (2010). Theory of carrier transport in bilayer graphene. Physical Review B, 81, 161407.

Das Sarma, S., Shaffique, A., Hwang, E. H., \& Rossi, E. (2011). Electronic transport in two-dimensional graphene. Reviews of Modern Physics, 83, 407-470.

Dinh, V. T., \& Nguyen, Q. K. (2013). Plasmon modes of double-layer graphene at finite temperature. Physica E: Low-dimensional Systems and Nanostructures, 54, 267272.

Dong, T. K. P., \& Nguyen, V. M. (2019). Plasmon modes in 3-layer graphene structures: Inhomogeneity effects. Physics Letters A, 383(33), 125971.

Hwang, E. H., \& Das Sarma, S. (2007). Dielectric function, screening, and plasmons in two-dimensional graphene. Physical Review B, 75, 205418.

Hwang, E. H., \& Das Sarma, S. (2009). Exotic plasmon modes of double layer graphene. Physical Review B, 80, 205405.

Jiang, B. -Y., Ni, G. -X., Addison, Z., Shi, J. K., Liu, X., Zhao, S. Y. F., Kim, P., Mele, E. J., Basov, D. N., \& Fogler, M. M. (2017). Plasmon reflections by topological electronic boundaries in bilayer graphene. Nano Letters, 17, 7080-7085.

Ju, L., Geng, B., Horng, J., Girit, C., Martin, M., Hao, Z., Bechtel, H. A., Liang, X., Zetti, A., Shen, Y. -R., \& Wang, F. (2011). Graphene plasmonics for tunable terahertz metamaterials. Nature Nanotechnology, 6(10), 630-634.

Lundeberg, M. B., Gao, Y., Woessner, A., Tan, C., Alonso-González, P., Watanabe, K., Taniguchi, T., Hone, J., Hillenbrand, R., \& Koppens, F. H. L. (2017). Thermoelectric detection and imaging of propagating graphene plasmons. Nature Materials, 16(2), 204-207.

Lv, M., \& Wan, S. (2010). Screening-induced transport at finite temperature in bilayer graphene. Physical Review B, 81, 195409.

Nguyen, V. M., \& Dong, T. K. P. (2018). Plasmon modes in bilayer-graphene-GaAs heterostructures including layer-thickness and exchange-correlation effects. International Journal of Modern Physics B, 32(23), 1850256. 
Nguyen, V. M., \& Dong, T. K. P. (2019). Plasmon modes in graphene GaAs heterostructures at finite temperature. International Journal of Modern Physics B, 33(16), 1950174.

Nguyen, V. M., \& Nguyen, Q. K. (2018). Plasmon modes in Dirac-Schrödinger hybrid electron systems including layer-thickness and exchange-correlation effects. Canadian Journal of Physics, 96(6), 615-621.

Nguyen, V. M., Nguyen, Q. K., \& Dong, T. K. P. (2019a). Plasmon modes in double bilayer graphene heterostructures. Solid State Communications, 294, 43-48.

Nguyen, V. M., Nguyen, Q. K., \& Dong, T. K. P. (2019b). Plasmon modes in MLG2DEG heterostructures: Temperature effects. Physics Letters A, 183(12), 13641370.

Nguyen, V. M., Nguyen, Q. K., \& Dong, T. K. P. (2019c). Plasmon modes in N-layer bilayer graphene structures. Solid State Communications, 298, 113647.

Nguyen, V. M., Nguyen, Q. K., \& Dong, T. K. P. (2020). Plasmon modes in doublelayer gapped graphene. Physica E: Low-dimensional Systems and Nanostructures, 118, 113859.

Nguyen, V. M., Nguyen, Q. K., \& Dong, T. K. P. (2021). Collective excitations in spinpolarized bilayer graphene. Journal of Physics: Condensed Matter, 33(10), 105301.

Ni, G. X., McLeod, A. S., Sun, Z., Wang, L., Xiong, L., Post, K. W., Sunku, S. S, Jiang, B. -J., Hone, J., Dean, C. R., Fogler, M. M., \& Basov, D. N. (2018). Fundamental limits of graphene plasmonics. Nature, 557, 530-533.

Ni, G. X., Wang, L., Goldflam, M. D., Wagner, M., Fei, Z., McLeod, A. S., Liu, M. K., Kellmann, F., Ozyilmaz, B., Castro-Neto, A. H., Hone, J., Fogler, M. M., \& Basov, D. N. (2016). Ultrafast optical switching of infrared plasmon polaritons in high-mobility graphene. Nature Photonics, 10, 244-247.

Patel, D. K. (2015). Transport properties of monolayer and bilayer graphene [Doctoral dissertation, Maharaja Sayajirao University of Baroda, India].

Politano, A., Argurio, P., Profio, G. D., Sanna, V., Cupolillo, A., Chakraborty, S., Arafat, H. A., \& Curcio, E. (2017). Photothermal membrane distillation for seawater desalination. Advanced Materials, 29(2), 1603504.

Politano, A., Cupolillo, A., Profio, G. D., Arafat, H. A., Chiarello, G., \& Curcio, E. (2016). When plasmonics meets membrane technology. Journal of Physics: Condensed Matter, 28(36), 363003.

Ramezanali, M. R., Vazifeh, M. M., Asgari, R., Polini, M., \& MacDonald, A. H. (2009). Finite-temperature screening and the specific heat of doped graphene sheets. Journal of Physics A: Mathematical and Theoretical, 42, 214015.

Sensarma, R., Hwang, E. H., \& Das Sarma, S. (2010). Dynamic screening and low energy collective modes in bilayer graphene. Physical Review B, 82, 195428. 
Sunku, S. S., Ni, G. X., Jiang, B. Y., Yoo, H., Sternbach, A., McLeod, A. S., Stauber, T., Xiong, L., Taniguchi, T., Watanabe, K., Kim, P., Fogler, M. M., \& Basov, D. N. (2018). Photonic crystals for nano-light in moiré graphene superlattices. Science, 362(6419), 1153-1156.

Vazifehshenas, T., Amlaki, T., Farmanbar, M., \& Parhizgar, F. (2010). Temperature effect on plasmon dispersions in double-layer graphene systems. Physics Letters A, 374(48), 4899-4903.

Wachsmuth, P., Hambach, R., Benner, G., \& Kaiser, U. (2014). Plasmon bands in multilayer graphene. Physical Review B, 90, 235434.

Wang, X. -F., \& Chakraborty, T. (2007). Coulomb screening and collective excitations in a graphene bilayer. Physical Review B, 75, 041404(R).

Yan, H., Li, X., Chandra, B., Tulevski, G., Wu, Y., Freitag, M., Zhu, W., Avouris, P., \& Xia, F. (2012). Tunable infrared plasmonic devices using graphene/insulator stacks. Nature Nanotech, 7(5), 330-334.

Zhu, J. -J., Badalyan, S. M., \& Peeters, F. M. (2013). Plasmonic excitations in Coulomb-coupled N-layer graphene structures. Physical Review B, 87, 085401. 